\title{
Communication and Ranging System for the Kepler Laboratory Demonstration
}

\author{
Raphael Wolf, German Aerospace Center (DLR) \\ Janis Surof, German Aerospace Center (DLR) \\ Juraj Poliak, German Aerospace Center (DLR) \\ Ludwig Blümel, German Aerospace Center (DLR) \\ Laura Agazzi, German Aerospace Center (DLR) \\ Ramon Mata Calvo, German Aerospace Center (DLR)
}

\section{BIOGRAPHIES}

Raphael Wolf studied electrical engineering at the Munich University of Applied Sciences. In 2018 he obtained his Masters and joined the Institute of Communications and Navigation at the German Aerospace Center (DLR) as a research associate. His main field of interest is hardware development, primary on FPGAs, for optical free-space communication and ranging applications.

Janis Surof studied electrical engineering at the technical University of Munich. He completed his Masters in 2016. Since 2017, he is scientific assistant at the Institute of Communications and Navigation at the German Aerospace Center (DLR). His fields of interests are optical coherent applications for free-space transmission for communications and optical ranging.

Juraj Poliak studied applied physics - astrophysics at Masaryk University in Brno, where he completed his Bachelor in 2010. This was parallel to his main study of electronics and communications at the Brno University of Technology, where he completed his Master in 2011 and his PhD in 2014. Since 2014 he works as Scientific Staff at the Institute of Communications and Navigation at the German Aerospace Center (DLR). His main topics of interest include satellite communication systems, broadband optical communication systems and design and verification of optical terminals for communications and time-frequency transfer.

Ludwig Bluemel received his diploma degree in microsystems engineering from the University of Applied Sciences in Munich in 2005 and his diploma degree in physics from the Ludwig-Maximilians-University in Munich in 2013. Since 2019 he works as Scientific Staff at the Institute of Communications and Navigation at the German Aerospace Center (DLR). His fields of interest are optical clocks, time references and optical frequency combs.

Laura Agazzi studied solid-state physics at the University of Pavia, completing her Master in 2007. She received her PhD in 2012 at the University of Twente, and worked in industry, at Montfort Laser and Menlo Systems. Since 2020 she works as Scientific Staff at the Galileo Competence Center at the German Aerospace Center (DLR). Her main fields of interest are clock technologies and frequency combs.

Ramon Mata Calvo studied telecommunications engineering at the Technical University of Catalonia and at the Technical University of Turin. He completed his Master in 2005 and his PhD in 2009 at the Technical University of Turin. Since 2010 he works at the Institute of Communications and Navigation at the German Aerospace Center (DLR). Since 2013 he is leading the group Advanced Optical Technologies, which deals with free-space optical satellite communications. His fields of interest are atmospheric turbulence, adaptive optics and time-frequency transfer by optical links. 


\begin{abstract}
Introducing optical inter-satellite links in a global navigation satellite system enhances orbit determination and offer frequency synchronization across the constellation. To show proof-of-concept and system capabilities a laboratory demonstrator has been developed and first tests in laboratory environment have been taken out. The demonstrator is buildup of two reciprocal units interconnected via bi-directional free-space optical links. Each unit is composed of opto-mechanical terminal, optical communication system and digital signal processing implemented on a field programmable gate array. Ranging and time transfer is realized utilizing a 25.55 Gigachip-per-second binary phase shift keying modulation scheme. Correlating the received ranging sequence with a local generated reference, pseudo range determination within the order of $100 \mu \mathrm{m}$ accuracy is achieved. In addition, a 50 Megabit-persecond data signal is multiplexed onto the ranging sequence for exchanging satellite and time information as well as user data. Frequency transfer is realized using a cavity stabilized laser as carrier transferring its stability onto the phase locked local oscillator at the receiver side. Global synchronization at system level is achieved by means of synchronizing the optical carrier and spread sequence to a highly stable clock reference as well precise ranging and information distributed through the data channel. Using onsatellite ultra-stable optical oscillator and a frequency comb for radio frequency reference generation short-term frequency stability in the order of $10^{-15} \mathrm{~s} / \mathrm{s}$ (Allan deviation at $1 \mathrm{~s}$ gate time) is achieved. The aim of this paper is to show the current status of the laboratory demonstrator development and present first measurements of the entirely setup system. A general overview of the digital signal processing is given and data transmission as well as time transfer are discussed in particular. The laboratory demonstrator setup and mechanical construction is presented. Clock stability transfer between high-accuracy radio frequency reference and the digital signal processing system is shown. Further, optical frequency transfer experiments are performed demonstrating successful synchronization between laboratories.
\end{abstract}

\title{
INTRODUCTION
}

Current global navigation satellite system (GNSS) is based on ultra-stable atomic clocks providing the reference for orbit and clock determination. The achievable accuracy is depended on the degree of synchronization which is limited by pseudo range calculation and underlying modelling assumptions. In [1] a novel GNSS, the Kepler system, is proposed introducing optical frequency references in particular cavity-stabilized lasers offering short-term frequency stability in the order of $10^{-15} \mathrm{~s} / \mathrm{s}$ at $1 \mathrm{~s}$ gate time. The envisioned constellation of 24 medium earth orbit and four to six low earth orbit satellites is interconnected by bidirectional optical inter-satellite links (OISL) transferring the optical reference stability allowing for frequency and time synchronization at system level. Further, OISL enable high accuracy ranging and communication across the constellation. Involved key technology being OISL, used for many years in space, as well as cavity-stabilized lasers successfully deployed since May $22^{\text {nd }}$, 2018 within the framework of the GRACE-Follow-On mission [2]. First simulation results for the Kepler constellation have been published achieving orbit determination accuracy in the centimeter range for position and tenth of micrometer-per-second for velocity with only one ground station being present [3]. An overview on current technical developments for OISL as well as optical oscillators for the proposed GNSS architecture is given in [4]. In order to validate the proposed concept of optical clock references and OISL a laboratory

demonstrator is developed. The setup is organized in two reciprocal units representing one satellite each. Each unit is composed of optical terminal, discussed more into detail in [5], as well as the communication and ranging system, shown in [6] and further discussed here.

The paper is organized in two main sections. First the development of laboratory demonstrator with focus on the digital signal processing system for ranging, data transmission and time transfer is presented. A brief insight on the system integration status and setup is given. The second part concentrates on performed measurements. In particular, the stability transfer in between a highaccuracy radio-frequency (RF) reference and the digital signal processing (DSP) unit respectively ranging and data transmitter is shown. The optical reference generation and distribution is validated ensuring proper operation and providing the stability baseline for all upcoming experiments. Further, successful frequency transfer between cavity-stabilized laser interconnected with the communication and ranging system via $60 \mathrm{~m}$ optical fiber is demonstrated. 


\section{LABORATOY DEMONSTATOR}

To verify the proposed concept of OISLs for ranging and data transmission within the framework of the Kepler constellation a laboratory demonstrator is build. In order to fully examine two-way OISL the setup is constituted of two reciprocal units representing one satellite respectively. Figure 1 shows the simplified block diagram of the laboratory demonstrator setup, for clarification subblocks establishing a one-directional link are highlighted and further explained. The DSP unit at the transmitter (Figure 1 left) multiplexes the pseudo random noise (PRN) based ranging sequence with the data signal. Following the spread sequence binary phase shift keying modulates the cavity stabilized laser and is send through the optical terminal towards the receiving unit. Here, the incoming light is coupled into the fiber by means of coarse-pointing assembly and fine-steering mirror. The optical signal is coherently mixed with the local oscillator (LO) and transferred to electrical domain by balanced photo-detectors. To match the incoming carrier's frequency and phase, an optical phase locked loop (OPLL) locks the LO phase onto the incoming carrier. Besides, it compensates for frequency Doppler and laser phase noise realizing frequency transfer between the units. Time Doppler is compensated for by the delay locked loop (DLL) by maximizing the correlation between the received ranging sequence and a locally generated reference and thereby allows for an absolute distance measurement between the units. A general overview of the scenario, the laboratory demonstrator as well as a detailed description of the implemented opto-mechanics and the coherent receiver is given in [5]. In the following the DSP system for communication and ranging is further explained and insights on the actual buildup of the laboratory demonstrator are given.

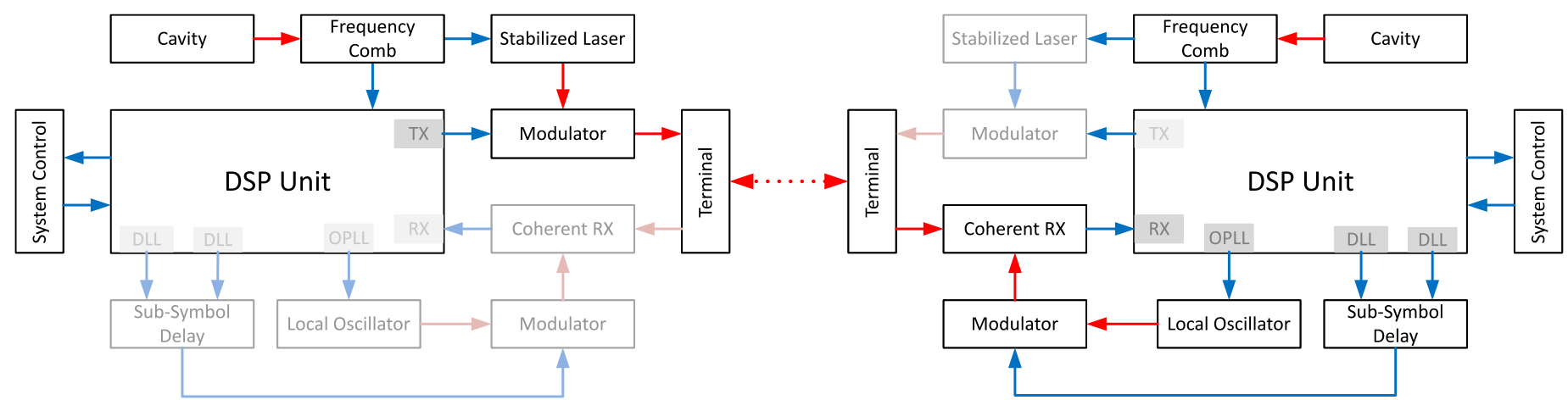

Figure 1: Block diagram of the communication and ranging system - sub-blocks utilized for one-directional link from left to right unit highlighted. Blue arrows represent electrical interconnects. The red dashed arrow illustrates free space optical link and red solid arrows represent fiber interconnects.

\section{Digital signal processing system}

The main requirement for the DSP system is to provide precise ranging and transfer the stability of the highly stable clock reference. Field programmable gate array (FPGA) technology satisfies these requirements allowing for high chip rate ranging sequence generation and derived clock design while maintaining flexibility in the development stage of a laboratory demonstrator. The simplified internal structure of the FPGA (also noted as DSP unit) and the interfaces with the frontend are shown in Figure 2. The main system clock is provided by a high precision phase locked loop (PLL) locked on the frequency comb which is referenced to the optical cavity. Hereby, the DSP is synchronized on the frequency comb transferring its stability onto the data and ranging generation and evaluation. To meet the desired ranging accuracy of $100 \mu \mathrm{m}$ the spread as well as the reference sequence are output at 25.55 Gigachip-per-second using multi-gigabit transceivers. Further DSP interfaces are given by the coherent receiver, sub-symbol delay and local oscillator control which are realized by analog-to-digital respectively digital-to-analog converters. For system configuration and monitoring a controller area network interface running CANopen protocol is implemented fitting in well with the remaining laboratory demonstrator setup. In order to perform ranging and data reception first the DLL is closed by maximizing the correlation of the received ranging sequence with the local generated reference. Hereby the received electrical signal power is optimized allowing the OPLL to lock on the incoming 50 Megabit-per-second data signal. The OPLL detector and loop filter are realized fully digital as part of the DSP. The local ranging reference is tunable down to sub-chip-shift level enabling ranging determination with accuracy in the order of $100 \mu \mathrm{m}$. The maxima determination relies on analogue correlation and observing three discrete points (early/middle/late) on the correlation curve in a sequential manner, a detailed description on the DLL implementation as given in [6]. 


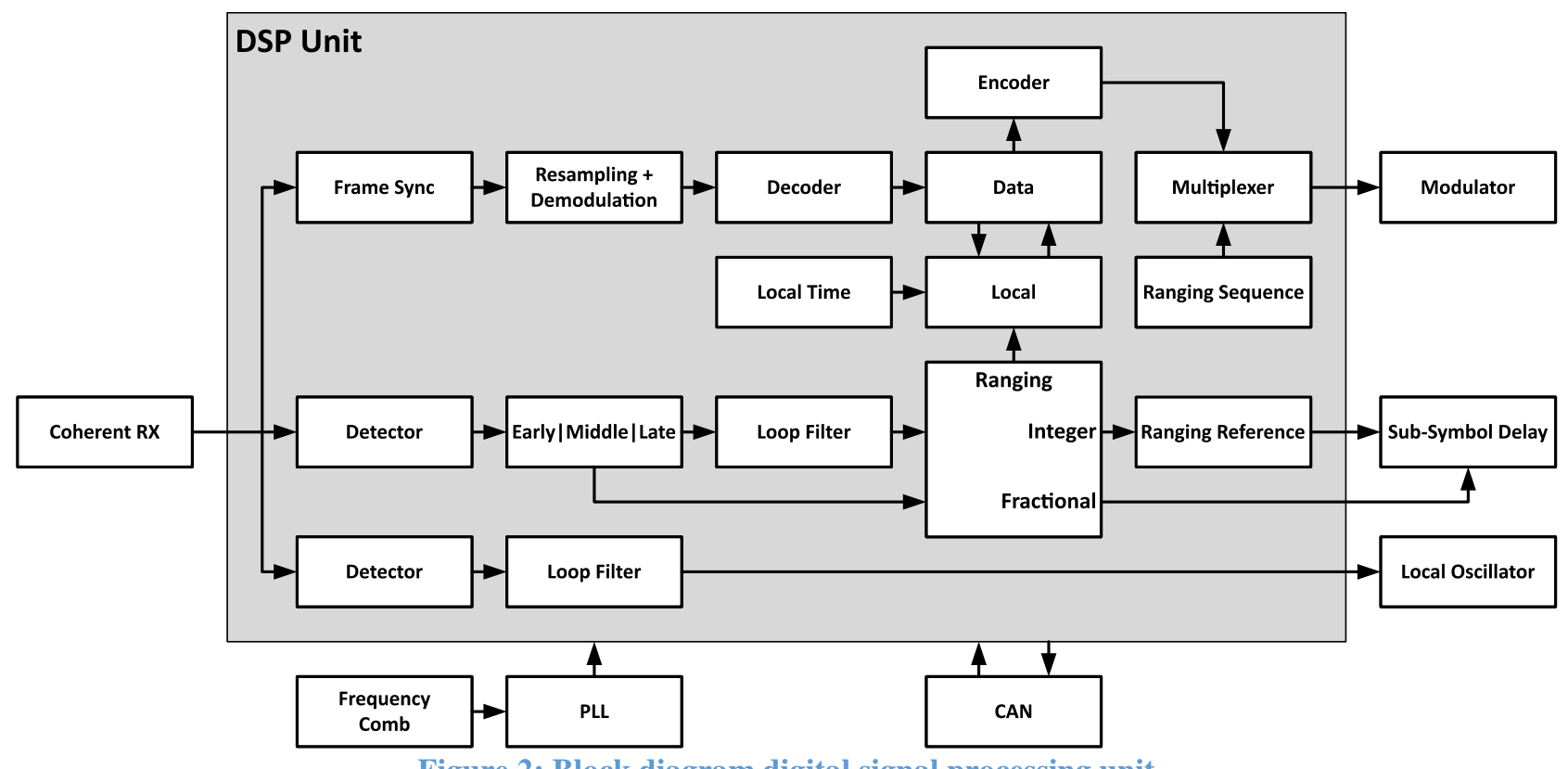

Figure 2: Block diagram digital signal processing unit

To exchange data a streamlined data transmission system has been developed. After coherent reception the incoming signal is sampled, digitized and forwarded to the DSP stage. Frame detection and timing recovery is based on autocorrelation with the leading synchronization header, "Frame Sync"-block in Figure 2. Subsequently resampling to 50 Megabit-per-second data rate and demodulation by means of hard decision takes place. In order to ensure sufficient data transitions for the OPLL to lock on as well as minimizing DC wander a line coding scheme is used which is removed by the decoder. The frame is decomposed and the plain data is written to random access memory. Memory management, frame de-/composition and flow control is handled by the "Data"-block. Figure 3 shows the currently implemented frame layout. System status is used to exchange general system information such as locking status of loops. Time stamp and time-of-flight (TOF) are exchanged in order to perform time transfer which is further explained in the following. The user data section of the frame allows for exchange of information like telemetry data or commands across the constellation. For demonstration purposes a PRN bit sequence is used to emulate this data.

\begin{tabular}{|l|l|l|l|l|}
\hline Sync Header & System Status & Time Stamp & Time Of Flight & User Data \\
\hline
\end{tabular}

Figure 3: Composition data frame

Another task of the communication and ranging system is to synchronize the satellite time scales across the constellation. Each unit independently generates its own local time derived from their highly stable clock reference. In order to harmonize the time scales amongst the units their mismatch is determined and corrected for. Figure 4 shows a simplified model for two-way transfer scheme and corresponding time contribution. Under the assumption that both clock references are equally stable the absolute mismatch between units can assumed to be static. Further assuming identical construction and quasi static thermal conditions over short time the transmitter and receiver introduced contributions are canceled out for two-way transfer. Given those boundaries the time mismatch can be determined by means of conveyed time stamps and TOF. TOF is retrieved through high precision ranging and conversion to corresponding timespan. The simplified time mismatch is given by:

$$
\varepsilon=\frac{\left(\tau_{T 0}+\tau_{R 0}\right)-\left(\tau_{T 1}+\tau_{R 0}\right)+\left(\Delta_{01}-\Delta_{10}\right)}{2} ; \varepsilon=\left|\varepsilon_{01}\right| \approx\left|\varepsilon_{10}\right|, \delta_{T X 0} \approx \delta_{T X 1}, \delta_{R X 0} \approx \delta_{R X 1}
$$

Within the framework of the laboratory demonstrator the time transfer is implemented as part of the DSP by "Local Time"- and "Time"-block, shown in Figure 2. Time-of-arrival (TOA) $\tau_{R 0}$ and $\tau_{R 1}$ is taken at the leading edge of the incoming data frame. Using 
a deterministic DSP implementation scheme time-of-transmission $\tau_{\mathrm{T} 0}$ and $\tau_{\mathrm{T} 1}$ is rigidly bound to TOA resulting in only one time stamp and the TOF $\Delta_{01}$ and $\Delta_{10}$ have to be exchanged for mismatch determination. Due to the static nature of the laboratory setup dynamic TOF behavior is modeled by introducing superimposed offset into the DLL control simulating one dimensional movement of the units.

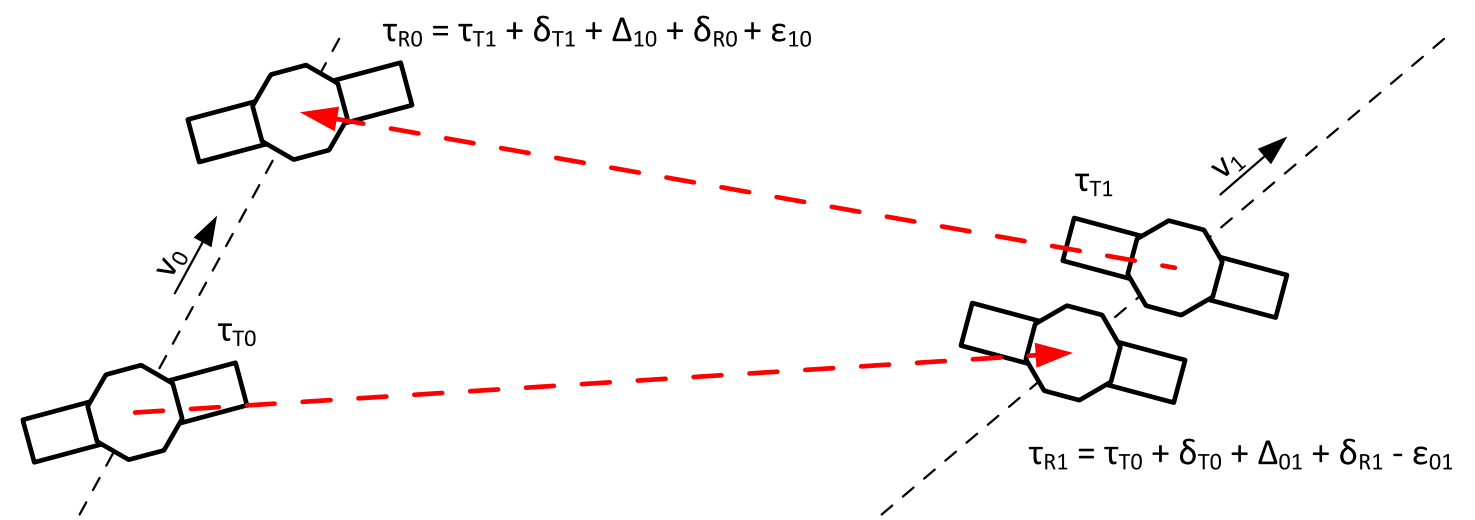

Figure 4: Two-way time transfer $-\tau_{\mathrm{T} 0} / \tau_{\mathrm{T} 1}$ transmit time stamp, $\tau_{\mathrm{R} 0} / \tau_{\mathrm{R} 1}$ receive time stamp, $\delta_{\mathrm{T} 0} / \delta_{\mathrm{T} 1}$ transmitter delay, $\delta_{\mathrm{R} 0} / \delta_{\mathrm{R} 1}$ receiver delay, $\Delta_{01} / \Delta_{10}$ time of flight, $\varepsilon_{01} / \varepsilon_{10}$ time offset and $v_{0} / v_{1}$ vehicle velocity

\section{System integration}

The laboratory demonstrator is organized into optical terminal integrated on an optical bench and a rack unit housing the communication and ranging system amongst others, both are shown in Figure 5. The optical terminal is built from commercial of the shelf (COTS) optics and mechanics as well as in-house developed electronics. The coherent bidirectional ranging unit (CoBRa) is internally organized into shelves housing the optical communication system as well as the DSP unit. All main components such as modulators, laser, coherent receiver and amplifiers are also COTS. The DSP unit is realized by means of a FPGA development platform providing multiple extension slots populated with in-house developed expansion boards realizing required interfaces and analogue electronics. The high accuracy frequency reference is provided by an ultra-low expansion spacer based optical reference laser systems (ORS) type ORS1500 and frequency comb type FC1500-250-ULN from Menlo Systems GmbH.
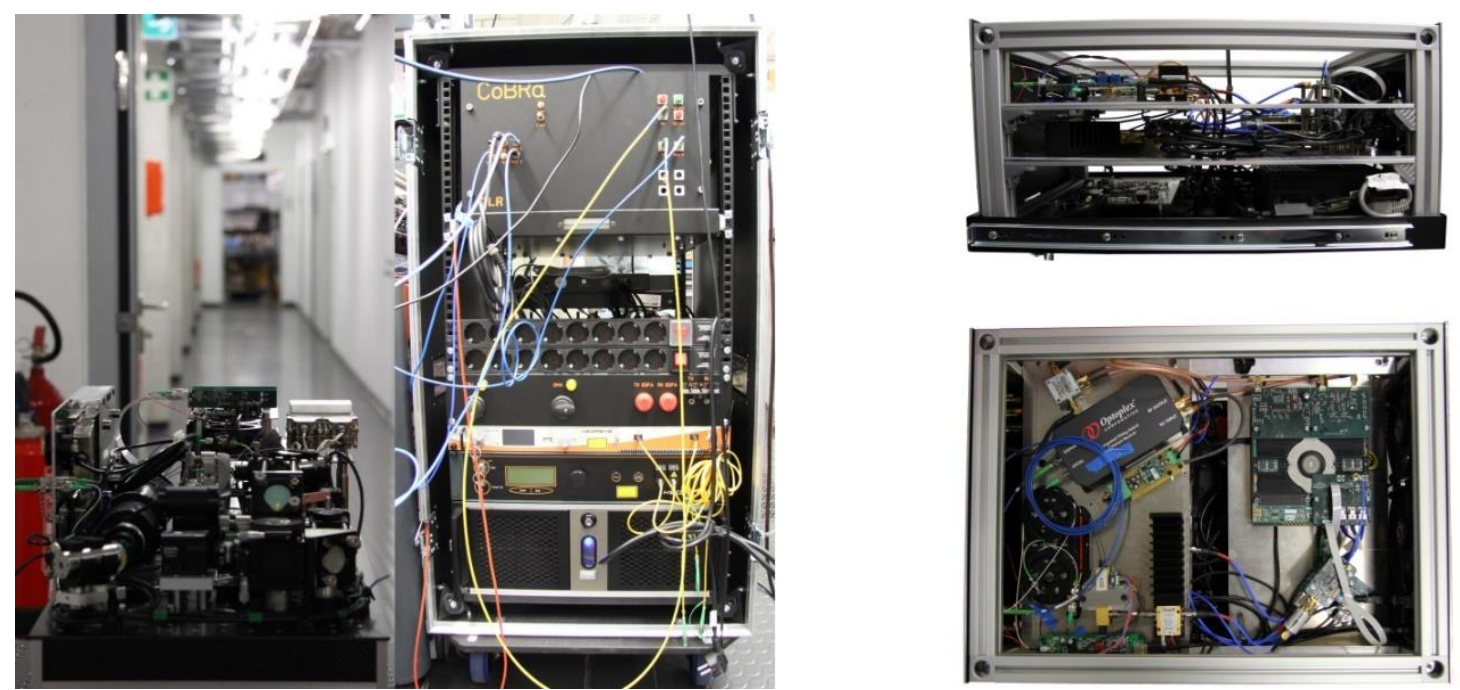

Figure 5: Optical terminal and rack unit (left picture) housing i.a. CoBRa (right picture detail view side and top) 


\section{MEASUREMENTS}

The integration of the laboratory demonstrator is far advanced allowing for full system operation and systematic verification. Evolving towards two-way communication and ranging over optical free space link operation baseline measurements are performed qualifying fundamental system aspects. In the following the stability transfer of a radio frequency clock reference onto the DSP system and thereby onto the ranging and time transfer is verified. Further, the optical frequency reference distribution between laboratories is qualified and first optical frequency transfer experiments between frequency reference and DSP unit over $60 \mathrm{~m}$ fiber are taken out. Quantifying the stability, the Allen deviation [7] is consulted.

\section{Radio frequency reference distribution}

The performance of time transfer and ranging strongly depends on the clock reference and additional timing jitter the FPGAs and other electronic components may introduce. Any deviation from the stable RF clock source directly decreases the precision of the measurements. To verify that the proposed system is capable of reaching the desired precision an experiment with a single DSP system is setup like shown in Figure 6. For this experiment the DSP system is referenced to a $10 \mathrm{MHz}$ output of a highly stable radio frequency reference, Kvarz CH1-75A active hydrogen maser (AHM), with a short-term stability better than $2 * 10^{-13} \mathrm{~s} / \mathrm{s}$ at $1 \mathrm{~s}$ gate time and an approximately $1 / \tau$ behavior to $10^{4} \mathrm{~s}$. The RF outputs of the frequency comb will as well provide a $10 \mathrm{MHz}$ signal. To satisfy the $100 \mathrm{MHz}$ digital input requirement of the DSP a high precision PLL multiplies the $10 \mathrm{MHz}$ to $100 \mathrm{MHz}$. The $100 \mathrm{MHz}$ output of the PLL and the $100 \mathrm{MHz}$ output of the AHM are compared against each other with a precision frequency comparator Vremya VCH-314. For input level adaption of the PLL signal a Femto HAS-X low noise amplifier is used. The overlapping Allan deviation of this common mode measurement is shown in Figure 6 on the right (blue, solid line). The performance of the AHM during this measurement is verified by comparing it to another AHM (blue, dashed). In a second experiment the transmitter output of the DSP generates the $100 \mathrm{MHz}$ clock signal. It is compared to the $100 \mathrm{MHz}$ AHM output and the overlapping Allan deviation is plot in Figure 6 on the right (red, line with circles). The AHM performance is measured with the second AHM (red, dotted line). Both AHM performance measurements show the same Allan deviation with a short-term stability of $2 * 10^{-13} \mathrm{~s} / \mathrm{s}$ at $1 \mathrm{~s}$ gate time which verifies proper operation. The PLL and the DSP unit transmitter show an approximately $1 / \tau$ behavior with a small additional noise penalty at $1 \mathrm{~s}$ gate time for the transmitter output due to FPGA internal clock distribution, digital processing and analogue frontend. Still the performance with a short-term stability of $1.3^{*} 10^{-13} \mathrm{~s} / \mathrm{s}$ at $1 \mathrm{~s}$ gate time indicates that the system is capable of reaching the desired precision in ranging and time transfer.
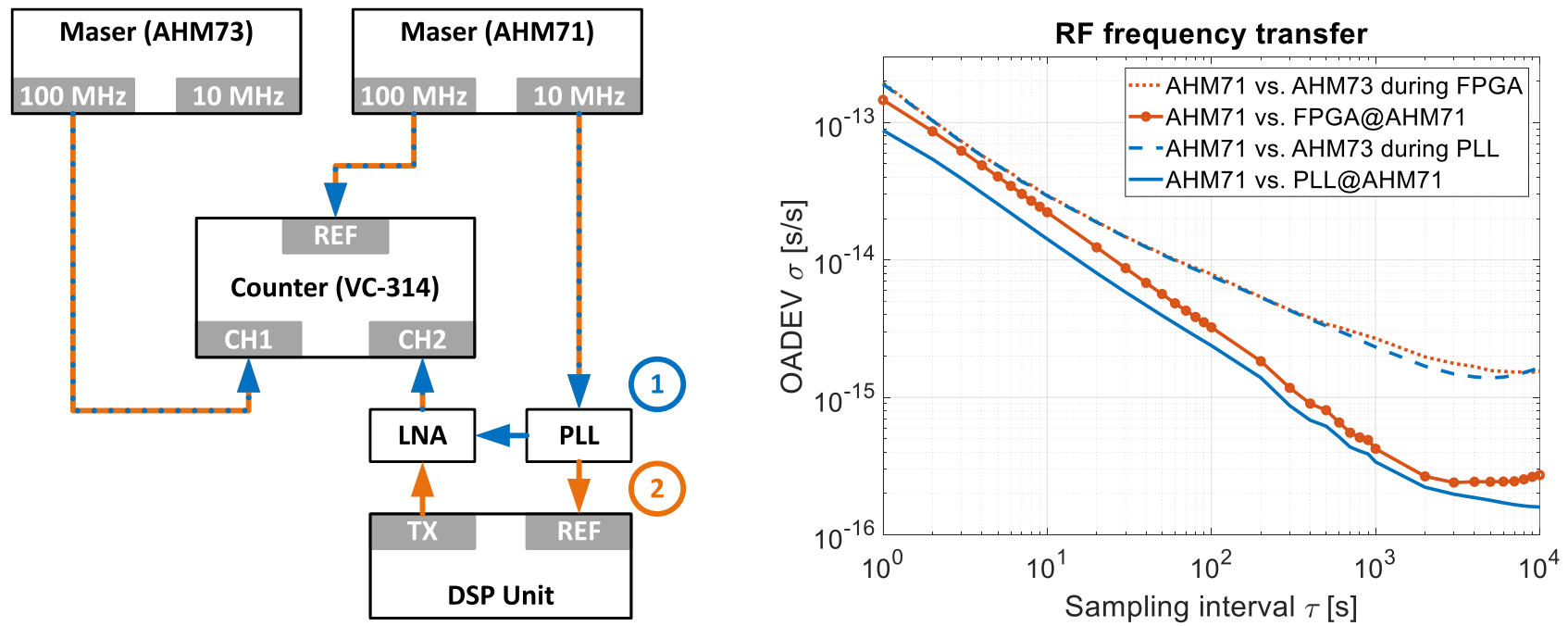

Figure 6: RF stability transfer measurement setup (left) and overlapping Allen deviation of the measurements (right). First measurement (AHM vs. PLL) in blue and second (AHIM vs. DSP unit) in red. Dashed blue-red arrows in the setup (left) are valid interconnects for both performed measurements. 


\section{Optical reference distribution and frequency transfer}

In the current development the setup for the frequency transfer is finalized. As shown in Figure 7 both sides of the laboratory demonstrator use optical references. The optical reference systems show typically a sub-Hz linewidth and a short-term stability of about $1 * 10^{-15} \mathrm{~s} / \mathrm{s}$ at $1 \mathrm{~s}$ gate time. The cavities have a linear frequency drift of about $1 \mathrm{kHz}$ per hour. The wavelengths are chosen at $1540.56 \mathrm{~nm}$ and $1564.68 \mathrm{~nm}$. This allows to use the cavities as carrier for the transmission. For RF clock generation and optical frequency measurements frequency combs are used which are referenced to the respective cavity respectively ORS by splitting parts of the light and locking on the heterodyne beat note between the nearest frequency comb mode and the cavity. Further it is possible to lock or compare additional optical sources to the referenced comb. The cavity and comb pairs are located in two laboratories with a $30 \mathrm{~m}$ hallway in between where the free space experiments will be executed. The laboratories are additionally connected by $60 \mathrm{~m}$ optical fibers to allow reference measurements. In [6] the frequency transfer of a reduced system with a cavity stabilized laser, frequency comb and a rubidium clock is presented. The results already proved the feasibility of the optical frequency transfer with an OPLL. The common mode measurement reached a stability of $1 * 10^{-15} \mathrm{~s} / \mathrm{s}$ at $1 \mathrm{~s}$ gate time. With the full setup being built, initial performance measurements of optical frequency transfer between the optical references are executed. These baseline measurements of the laboratory optical reference distribution are conducted in order to ensure frequency stability other entities of the setup are referenced to.

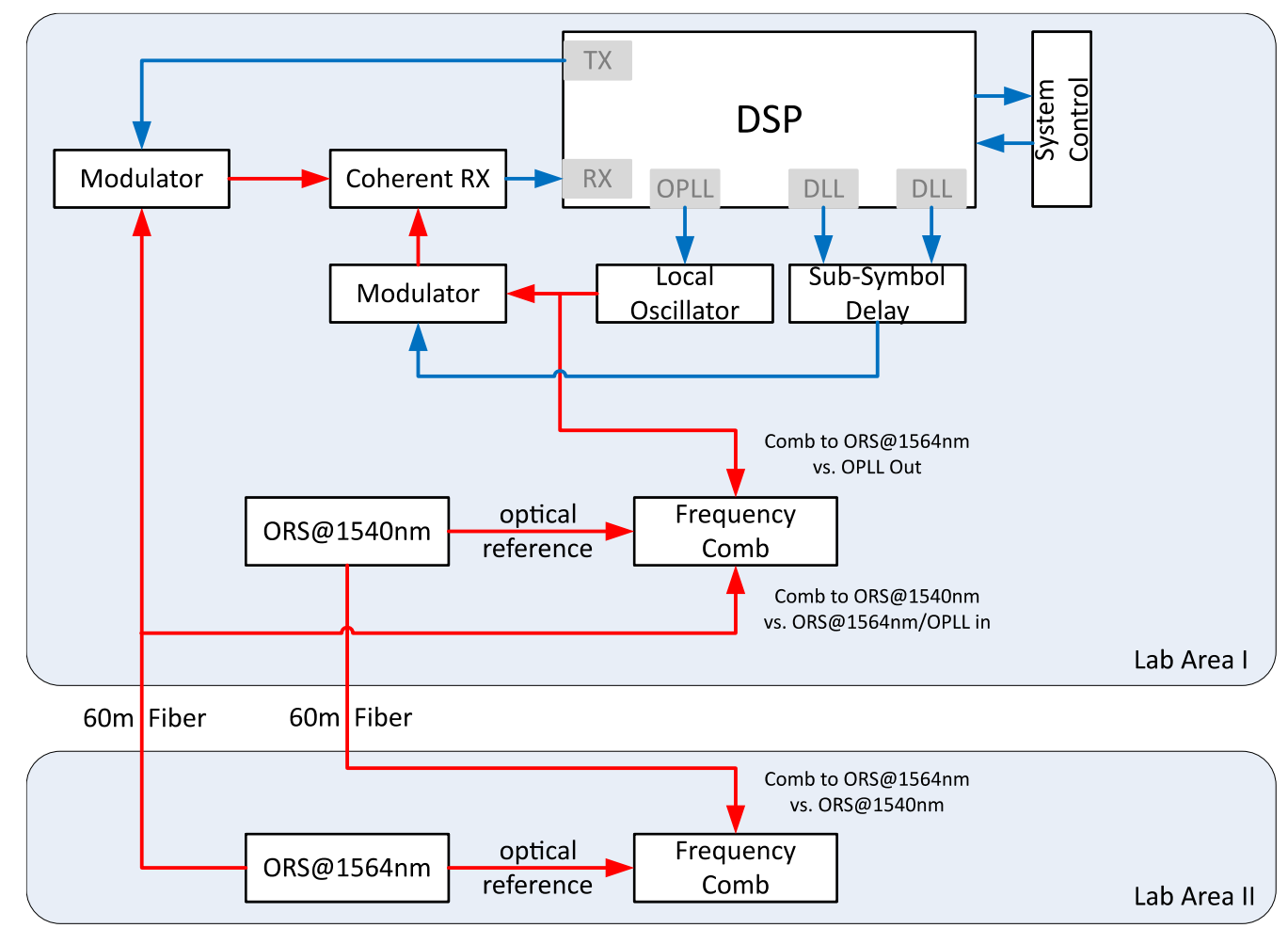

Figure 7: Setup for optical frequency transfer verification and OPLL short term stability evaluation

The performance of the cavities and combs is evaluated by measuring each cavity against the opposite frequency comb. A direct comparison between the cavities is not possible as the wavelengths are too far apart for a direct beat node measurement. Referencing the combs to their respective cavity their stability is transferred onto the combs. In Figure 8 the Allan deviation of the comparison measurements between the frequency comb referenced to ORS at $1564 \mathrm{~nm}$ and the ORS at $1540 \mathrm{~nm}$ (blue, dashed) as well as the frequency comb referenced to ORS at $1540 \mathrm{~nm}$ and the ORS at $1564 \mathrm{~nm}$ (red, dashed) are shown. As expected, both are overlapping within their error bars. It shows that the referencing of the combs to the cavities is working properly. The single stability of the ORS can be estimated by a factor of $1 / \sqrt{ } 2$ lower than the measured system stability to $1.3^{*} 10^{-15} \mathrm{~s} / \mathrm{s}$ at $1 \mathrm{~s}$ gate time.

To evaluate the stability of the local oscillator of the OPLL, a second $60 \mathrm{~m}$ fiber with $1564 \mathrm{~nm}$ is routed to the opposite laboratory. There it is modulated with the PRN sequence and multiplexed data, provided by the receiver DSP TX output for simplicity. Then, the beam is fed into the coherent receiver and mixed with the LO modulated with the PRN reference. After closing the DLL, the 
OPLL is locked on the incoming carrier. The LO which is locked on the $1564 \mathrm{~nm}$ ORS is split and a part of the beam is feed to the frequency comb. The frequency comb referenced to the ORS at $1540 \mathrm{~nm}$ is compared to the $1564 \mathrm{~nm}$ ORS signal and to the LO. The Allan deviations of these measurements are shown in Figure 8. The comparison with the ORS (yellow) shows the same system stability at $1 \mathrm{~s}$ gate time. After $10 \mathrm{~s}$ gate time the curve deviates from the base line measurements. Changes in the laboratory conditions, in this case of the temperature, lead to deviations in the drift rates of the two ORS and optical Doppler shift on the $60 \mathrm{~m}$ fiber connection. The Allen deviation of the LO (purple) has a slightly lower measured stability of $2.2^{*} 10^{-15} \mathrm{~s} / \mathrm{s}$ at $1 \mathrm{~s}$ gate time. At around $10 \mathrm{~s}$ gate time it converges to the parallel ORS measurement. Still the stability performance of the OPLL is capable to successfully transfer the incoming carrier stability on the LO. A calculated single stability of the OPLL is estimated to $1.05^{*} 10^{-15} \mathrm{~s} / \mathrm{s}$ at $1 \mathrm{~s}$ gate time and is below $1.2 * 10^{-15} \mathrm{~s} / \mathrm{s}$ up to $10 \mathrm{~s}$ gate time.

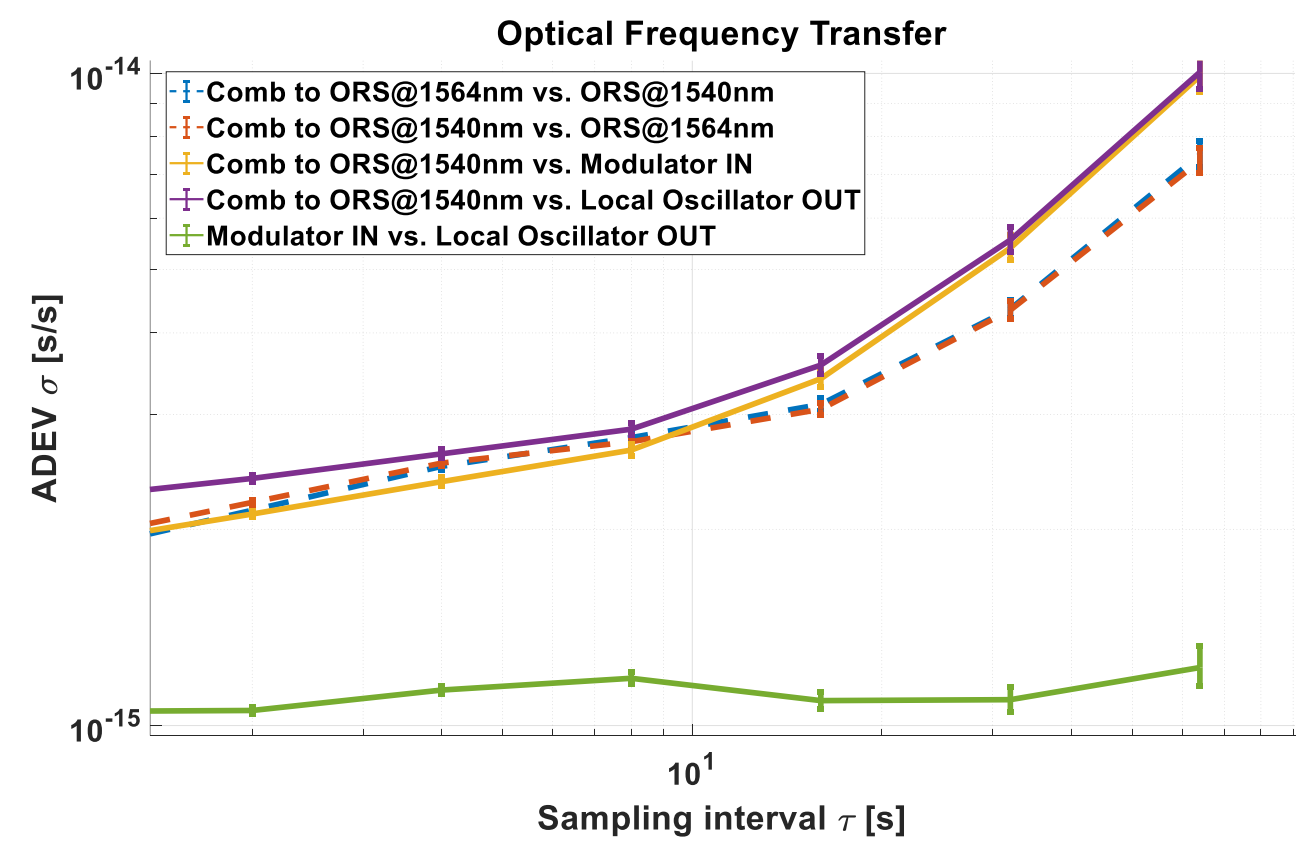

Figure 8: ADAV of the comparison between an optical reference system ORS1500 and the OPLL local oscillator output as well as a short-term stability of the optical reference systems ORS1500 in the setup

\section{CONCLUSION}

The current status of the laboratory demonstrator development for communication and ranging over OISL within the framework of the Kepler constellation is shown. The FPGA-based DSP unit is explained more in detail with focus on data transmission and time transfer. The integration of the laboratory demonstrator is primarily finished and set up on the $30 \mathrm{~m}$ free space test bed at the premises of DLR. The setup is composed of two reciprocal units each consisting of opto-mechanical terminal, optical communication and ranging system and high precision frequency reference. In preparation for full bi-directional free-space ranging, frequency transfer and data transmission different system aspects are tested and verified. The stability transfer of a high precision RF reference onto the DSP unit respectively the communication and ranging shows satisfying results with a short-term stability of $1.3 * 10^{-13} \mathrm{~s} / \mathrm{s}$ at $1 \mathrm{~s}$ gate time. Verification of the reference transformation from optical to RF and distribution for both sites ensures valid baseline for upcoming experiments. First frequency transfer experiments between ORS and spatially divided OPLL demonstrate successful frequency transfer maintaining stability with estimated OPLL stability of $1.05 * 10^{-15} \mathrm{~s} / \mathrm{s}$ at $1 \mathrm{~s}$ gate time. In the next steps the system is further qualified moving forward to demonstrate full bi-directional ranging, frequency and time transfer as well as communication over 30 m optical free space link. 


\section{ACKNOWLEDGMENTS}

This project is supported by the Helmholtz-Gemeinschaft Deutscher Forschungszentren e.V. under grant numbers ZT-0007 (ADVANTAGE, Advanced Technologies for Navigation and Geodesy). The project ADVANTAGE is a joint project of DLR and the Geo Forschungszenttum (GFZ) Postdam. It aims at defining a future system for navigation, geodesy and metrology.

\section{REFERENCES}

[1] C. Günther, "Kepler - Satellite Navigation without Clocks and Ground Infrastructure," in Proceedings of the 31st International Technical Meeting of The Satellite Division of the Institute of Navigation (ION GNSS+ 2018), 2018.

[2] K. Abich, A. Abramovici, B. Amparan, A. Baatzsch, B. B. Okihiro, D. C. Barr, M. P. Bize, C. Bogan, C. Braxmaier, M. J. Burke, K. C. Clark, C. Dahl, K. Dahl, K. Danzmann, M. A. Davis, G. de Vine, J. A. Dickson, S. Dubovitsky, A. Eckardt, T. Ester, G. F. Barranco, R. Flatscher, F. Flechtner, W. M. Folkner, S. Francis, M. S. Gilbert, F. Gilles, M. Gohlke, N. Grossard, B. Guenther, P. Hager, J. Hauden, F. Heine, G. Heinzel, M. Herding, M. Hinz, J. Howell, M. Katsumura, M. Kaufer, W. Klipstein, A. Koch, M. Kruger, K. Larsen, A. Lebeda, A. Lebeda, T. Leikert, C. C. Liebe, J. Liu, L. Lobmeyer, C. Mahrdt, T. Mangoldt, K. McKenzie, M. Misfeldt, P. R. Morton, V. Müller, A. T. Murray, D. J. Nguyen, K. Nicklaus, R. Pierce, J. A. Ravich, G. Reavis, J. Reiche, J. Sanjuan, D. Schütze, C. Seiter, D. Shaddock, B. Sheard, M. Sileo, R. Spero, G. Spiers, G. Stede, M. Stephens, A. Sutton, J. Trinh, K. Voss, D. Wang, R. T. Wang, B. Ware, H. Wegener, S. Windisch, C. Woodruff, B. Zender and M. Zimmermann, "In-Orbit Performance of the GRACE Follow-on Laser Ranging Interferometer," Physical Review Letters, vol. 123, 72019.

[3] G. Giorgi, B. Kroese and G. Michalak, "Future GNSS constellations with optical inter-satellite links. Preliminary space segment analyses," in 2019 IEEE Aerospace Conference, 2019.

[4] G. Giorgi, T. D. Schmidt, C. Trainotti, R. Mata-Calvo, C. Fuchs, M. M. Hoque, J. Berdermann, J. Furthner, C. Günther, T. Schuldt, J. Sanjuan, M. Gohlke, M. Oswald, C. Braxmaier, K. Balidakis, G. Dick, F. Flechtner, M. Ge, S. Glaser, R. König, G. Michalak, M. Murböck, M. Semmling and H. Schuh, "Advanced technologies for satellite navigation and geodesy," Advances in Space Research, vol. 64, p. 1256-1273, 92019.

[5] J. Poliak, R. M. Calvo, J. Surof, M. Richerzhagen and R. Wolf, "Laboratory Demonstrator of Optical Inter-satellite Links for the Kepler System," in Proceedings of the 31st International Technical Meeting of The Satellite Division of the Institute of Navigation (ION GNSS+ 2018), 2018.

[6] J. Surof, J. Poliak, R. M. Calvo, M. Richerzhagen, R. Wolf and T. Schmidt, "Laboratory Characterization of Optical Intersatellite Links for Future GNSS," in Proceedings of the 32nd International Technical Meeting of the Satellite Division of The Institute of Navigation (ION GNSS+ 2019), 2019.

[7] D. W. Allan, "Time and Frequency (Time-Domain) Characterization, Estimation, and Prediction of Precision Clocks and Oscillators," IEEE Transactions on Ultrasonics, Ferroelectrics and Frequency Control, vol. 34, p. 647-654, 111987. 